\title{
Развитие алгоритма спонтанного структурирования иифровых изображений природных комплексов
}

\author{
Э.Я. Островский 1, д.т.н., научный руководитель \\ C.B. Румяниев ${ }^{2}$, к.фр.-м.н., руководитель группы, Sergey_Rumyancev@srisa.ru \\ B.A. Фадеев 1, генеральный директор, cs-tehno@таil.ru
}

1 Компания Форес, 109518, г. Москва, Россия

2 Федеральный научный иентр Научно-исследовательский институт системных исследований РАH, 123182, г. Москва, Россия

Рассматривается совершенствование алгоритма спонтанного формирования непротиворечивой структуры на объектах цифровой матрицы с данными наблюдения параметров (магнитных, гравиметрических, радиоактивных и др.) исследуемого природного комплекса.

Приводится постановка задачи, рассматривается технология спонтанного структурирования, альтернативная традиционным технологиям, которая, выявляя стабильные свойства-признаки, создает непротиворечивую уникальную (единственно возможную) структуру.

Рассматривается алгоритм как последовательность ключевых этапов, составляющих основу технологии спонтанного формирования непротиворечивой структуры. Дается краткий обзор применения технологии.

Авторы подчеркивают роль человека как эксперта в использовании технологии спонтанного формирования непротиворечивой структуры. В заключении перечисляются возможные приложения технологии.

Таким образом, дано развитие технологии (концепция, алгоритм, ПО) формирования непротиворечивой структуры в виде совокупности непересекающихся устойчивых многомерных однородностей - ассоциаций по смежности. Последние - области адекватной экстраполяции (прогнозирования) для образующих их объектов.

Ключевые слова: прогноз, структуры, технология.

В трудах академика Дородницина А.А. при сравнении точных и описательных наук утверждается, что точность или описательность не являются свойствами тех или иных явлений природы, это лишь характеристика этапа развития наук, их изучающих [1]. Развитие вычислительной техники дало возможность многим наукам перейти из раздела описательных в точные. Геологоразведка в свое время основывалась именно на рецептах, выработанных практикой, на мнении экспертов [2-4]. В настоящей работе рассматривается развитие технологии анализа цифровых изображений. Начало этой технологии было положено именно в геологоразведке, которая, благодаря развитию вычислительной техники, получила оформление как точная наука [2-5].

Авторы статьи рассматривают дальнейшее развитие техники спонтанного структурирования применительно к матрицам цифровых изображений [6-8].

\section{Концепция спонтанного структурирования: самоорганизация хаоса в устойчивые однородности - части структуры}

Структура произвольной цифровой матрицы событий (digital matrix event, DME) - это совокупность непересекающихся множеств однородных объектов (однородностей), инструмент превращения DME в информацию об исследуемой реальности и прогнозирования ее объектов. В рамках данного определения очевидно существование струк- тур двух типов, обусловленных двумя типами однородностей.

1. Наделенные только им присущими свойствами-признаками (иифровое изображение - 2D3D-матрица событий, объектам (быть может, не всем) которой поставлены в соответствие циифровые значения (дискретные отсчеты)).

2. Наделенные только им присущими стабильными свойствами-признаками.

Традиционные технологии формируют на объектах DME первый тип однородностей и сколь угодно много разнообразных (противоречивых) структур в зависимости от той или иной субъективной предустановки конкретного эксперта (его знаний, опыта, интуиции, ожиданий, предпочтений и т.п.) $[9,10]$. В терминах зрительного восприятия такой предустановочный подход к структурированию означает: видеть известное или желаемое в неизвестном.

Подход в духе известного принципа дополнительности, постулирующего существование неустранимой множественности точек зрения на одну и ту же реальность (приниип дополнительности рассматривается как впервые введеное в квантовую физику Н. Бором обобщение соотношений неопределенности Гейзенберга). Однако он же становится причиной неадекватных структур - фантомов, артефактов типа слуховых или зрительных галлюцинаций. Разработанная технология спонтанного структурирования, альтернативная традиционным, выявляя однородности второго типа, создает непротиворечивую уникальную (един- 
ственно возможную) структуру. Если в качестве основного информационного признака любого изображения принять однородность, сообщество эквивалентно идентифицированных объектов, то любую DME можно представить хаосом однородностей, которые стохастически образуются на ней и ее функционально разнообразных проекциях $[11,12]$. Такие неравновесные изолированные системы спонтанно эволюционируют к стационарному состоянию, совместимому с наложенными граничными условиями (числом объектов, сетью наблюдений, наблюдаемыми параметрами и т.д.).

Индикаторами этого состояния являются скрытые в стохастическом шуме последовательности высококогерентных (взаимовложенных) однородностей, где каждая последующая вложена в предыдущую (является ее подмножеством с числом объектов $\geq 0.5$ числа объектов предыдущей). Эти высококогерентные однородности характеризуются минимальным производством энтропии и, следовательно, наиболее устойчивы, поэтому они могут использоваться в качестве стабильных признаков DME, ее специфичных многомерных идентификаторов. Эквивалентно идентифицированные ими объекты образуют ассоциации по смежности, поскольку для них справедливо утверждение: появление одного из объектов ассоциации в составе того или иного идентификатора означает появление в нем остальных объектов ассоциации. То есть объект DME может принадлежать одной и только одной ассоциации по смежности, которая в силу этого является областью адекватной пространственной экстраполяции (прогнозирования) для составляющих ее объектов.

\section{Алгоритм спонтанного формирования уникальной высокоинформативной структуры}

Спонтанное структурирование осуществляется приведенной далее последовательностью операций, названных авторами «р-алгоритм».

- Приведение исходных данных к формату цифровой матрицы. Отличия могут быть в машинном представлении значения элементов матрицы - integer*2, integer*4, real*4, real*8. Например, сейсмические данные формата SGY (формат чисел - плавающая запятая), аэро- или космоснимки формата цифрового изображения.

- Формирование матриц изображения в единый проект, определяемый размерами и заданием маски. Маска определяет точки измерений и формируется как двоичный файл. Использование маски позволяет проводить анализ на одних и тех же исходных данных, но отличающихся областью, выделенной посредством маски. Маска может также испоьзоваться для фильтрации исходных данных. Типичный пример - маскирование облаков на космоснимках.
- Увеличение степени разнообразия - добавление в проект матриц, зависящих от исходных данных. Необходимость этой операции возникает при проведении анализа по ограниченному количеству исходных данных. Практика применения алгоритма указывает на необходимость этого этапа. Примером могут служить сейсмические данные, радиолокационные и черно-белые изображения.

На этом этапе, как правило, используется алгоритм скользящего окна для расчета заданных пользователем функций. При этом необходимо учитывать неполное заполнение скользящего окна на границе отсуствия данных. В то же время настоящая технология использует скользящее окно как меру близости на пространстве точек наблюдения. На практике данные типа геохимического опробования, которые могут быть заданы на ограниченном множестве случайно раположенных точек, не дают использовать эту меру близости. Однако использование сегментации только исходных данных позволяет применять технологию и в этом случае.

- Формирование двоичных фильтров (сегментация изображения) - разделение цифровой матрицы на области (фрагменты), однородные по критерию, для которого сформирована цифровая матрица, в соответствии с условием $2 N<K<2 N+1$, где $K$ - число объектов цифровой матрицы; $N$ - число отбираемых для структурирования двоичных фильтров (рис. 1). В идеальном случае двоичный фильтр цифровой матрицы делит ее на равные по площади фрагменты.

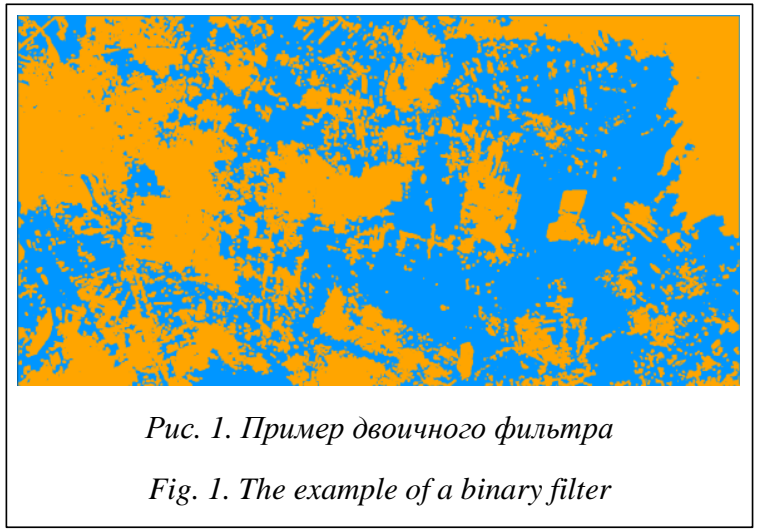

Пересечение всех цифровых фильтров дает пространство элементарных множеств (событий), объединение которых дает всю область анализа.

- Отбор двоичных фильтров. Количество цифровых фильтров определяется желаемой подробностью классификации анализируемой области. Необходимо иметь в виду, что найдутся такие пересечения двоичных фильтров, в которых нет ни одной точки данных. Отбор фильтров должен быть выполнен по сходству. Применение основного принципа (максимальная площадь и минимальная длина границы) имеет своей целью получить наиболее плотные по заполнению фильтры и избежать ситуаций типа шахматной доски. 
- Построение первичной классификации многообразий элементарных однородностей как пересечения выбранных двоичных фильтров. При программной реализации удобнее представить классификацию как кодовый файл, определяющий первичную классификацию.

- Выделение устойчивых однородностей для первичной классификации. Проводится на основе анализа последовательностей вложенных однородностей. Результаты такого анализа позволяют выделить последовательности, обладающие свойством устойчивости. Примечательно, что, несмотря на большое количество точек, устойчивых последовательностей оказывается несколько десятков. Этот примечательный факт и послужил основой технологии спонтанного структурирования.

- Идентификация объектов DME устойчивыми однородностями - построение ассоциации по смежности.

- Визуализация результатов - возможность графического представления объектов спонтанного структурирования:

- примитивная визуализация - раскраска цифровых матриц по цветовой шкале, пропорциональной значению или площади, занимаемой данным признаком, двухцветная раскраска области по двоичному фильтру или по пространству элементарных множеств, заданных пересечением нескольких двоичных фильтров;

- визуализация уникальной (непротиворечивой) высокоинформативной структуры DME - coвокупности непересекающихся ассоциаций по смежности;

- визуализация отдельных ассоциаций по смежности, содержащих объекты, заданные экспертом для прогнозирования.

\section{Опыт применения технологии спонтанного структурирования}

Опыт применения основан на развитии алгоритма для возможности применения к исходным данным различной природы. Изначально алгоритм строился применительно к анализу аэрогеофизических данных. В настоящее время допустим анализ сейсмических данных как 2D-формата, так и $3 \mathrm{D}$. Сложностью в трехмерном случае остается визуализация, но эта проблема не связана с алгоритмом и решается преобразованием результатов прогноза в формат программы 3D-визуализации (например, использование элементов типа voxel в VoxelGeo системе объемной визуализации и детальной интерпретации 3D-данных) (рис. 2) [13].

Для геофизических исследований эксплуатационных нефтегазовых скважин и для данных геофизического опробования необходим модифицированный алгоритм, не использующий меры близости точек исходных данных.

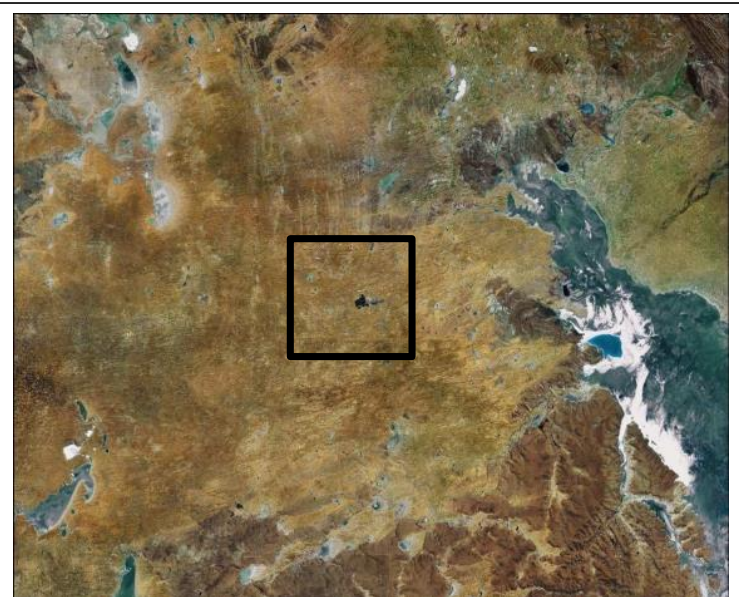

Pис. 2. Исходные данные - GoogleMap. В иентре снимка Olympic Dam Mine Cu-Au-U depositизвестное месторождение в Австралии

Fig. 2. Input data is GoogleMap. In the center of the picture is Olympic Dam Mine Cu-Au-U deposit, a well-known deposit in Australia

Отметим, что стремительное развитие вычислительной техники позволяет анализировать аэрокосмоснимки предельных размеров, но в то же время обеспечивает получение исходных данных с труднопредставимыми скоростями. Американская статистика свидетельствует о 9,5 млрд фотографий в день, сделанных только американцами, больше трети фотографий при этом снимается телефоном.

Ограничения на время для обработки одного снимка приводят, как следствие, к ограничению на размер исходных данных, которые могут возникнуть при анализе аэрокосмоснимков в режиме реального времени (рис. 3).

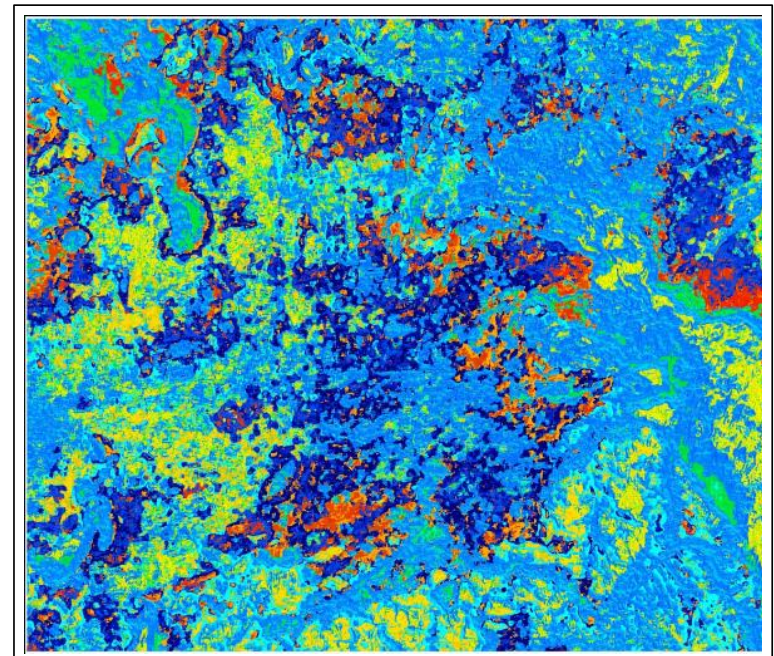

Рис. 3. Результат обработки - консистентная структура изображения на рисунке 1

Fig. 3. A result of processing. The consistent structure of the image in Figure 1 


\section{Человеческий фактор, или роль эксперта в использовании технологии}

Использование технологии, вообще говоря, не зависит от использования вычислительной техники, которая определяет только время, необходимое для анализа, или размеры анализируемой цифровой матрицы. Однако принципиальным является участие в проведении анализа эксперта, то есть развитие технологии рассматривается как создание инструмента, который используется экспертом для решающего заключения.

\section{Заключение}

В работе представлено развитие созданной ранее технологии (концепции, алгоритма) спонтанного структурирования произвольной цифровой матрицы, которая может эффективно использоваться для прогнозирования объектов, заданных в качестве целевых (поиск полезных ископаемых, участков развития болезнетворных процессов в тканях живого организма и др.), оценки природных ресурсов, мониторинга изменений во времени консистентной структуры (экологический мониторинг последствий хозяйственной деятельности и т.п.) и для усиления контрастности выявляемых однородностей.

Кроме того, технология применима при поиске следов упорядоченности (устойчивых однородностей) в шумовых средах (обнаружение и контроль загрязнений водной или воздушной среды, выявление слабоконтрастных структурных «ловушек» в земной коре и т.д.) и в атрибуции уникальных произведений искусства, в частности, на основе оценки сходства разных объектов.

\section{Литература}

1. Дородницын А.А., Каспшицкая М.Ф., Сергиенко И.В. Об одном подходе к формализации классификации // Кибернетика. 1976. № 6. С. 132.

2. Островский Э.Я. Радиоактивные аномалии как индикаторы рудного процесса // Докл. АН СССР. 1975. Т. 221. № 1. C. 201-204.

3. Портнов А.М. О геохимической связи калия и тория в изверженных породах и околорудных метасоматитах // Докл. АН СССР. 1978. Т. 239. № 6. С. 1441-1444.

4. Островский Э.Я., Портнов А.М. Индикаторные свойства геохимической связи калия и тория // Геохимия. 1979. № 5. C. $767-774$.

5. Портнов А.М. Радиогеохимический поиск руды // Природа. 1984. № 5. С. $99-105$.

6. Островский Э.Я., Румянцев С.В. Спонтанное структурирование цифровых геофизических изображений природных комплексов; прогнозирование полезных ископаемых // ДАН. Геофизика. 2004. Т. 398. № 3. С. 396-399.

7. Островский Э.Я., Румянцев С.В., Фадеев В.А. Новый структурный анализ цифровых изображений природных комплексов // Геопрофи. 2005. № 2. С. 49-51.

8. Островский Э.Я. Асимптотическое прогнозирование на основе дистанционных геофизических исследований // Докл. AH CCCP. 1986. T. 289. № 4. C. 827-836.

9. Гонсалес Р., Вудс Р. Цифровая обработка изображений. M.: Техносфера, 2005. $1072 \mathrm{c}$.

10. Гонсалес Р., Вудс Р., Эддинс С. Цифровая обработка изображений в среде MATLAB. М.: Техносфера, 2006. 621 с.

11. Гленсдорф П., Пригожин И. Термодинамическая теория структуры, устойчивости и флуктуации. М.: Мир, 1973. $280 \mathrm{c}$.

12. Федер Е. Фракталы. М.: Мир, 1991. 254 с.

13. VoxelGeo. Объемная интерпретация и выделение тел. URL: http://www.pdgm.com/products/voxelgeo/ (дата обращения: 02.04.18)

\section{Development of the spontaneous structuring algorithm of natural complex digital images}

E.Ya. Ostrovsky ${ }^{1}$, Dr.Sc. (Engineering), Scientific Supervisor

S.V.Rumyantsev ${ }^{2}$, Ph.D. (Physics and Mathematics), Head of Group, Sergey_Rumyancev@srisa.ru

V.A. Fadeev ${ }^{1}$, Director General, cs-tehno@mail.ru

${ }^{1}$ Fores Ltd., 109518, Moscow, Russian Federation

${ }^{2}$ Scientific Research Institute for System Analysis of the Russian Academy of Sciences, Moscow, 123182, Russian Federation

Abstract. The paper considers the way of improving the algorithm of spontaneous formation of a consistent structure on digital matrix objects with observation data of parameters (magnetic, gravimetric, radioactive etc.) of the natural complex under investigation.

In the section "A Spontaneous Structuring Concept: Chaos Self-Organization in Stable Homogeneities - Structure Parts" the authors state the problem. They consider a spontaneous structuring technique that is alternative to traditional technologies and reveals stable properties (signs), creates a unique (only possible) consistent structure.

In the section "The Algorithm of Spontaneous Formation of a Unique Highly Informative Structure" the authors consider the algorithm as a sequence of key stages that form the basis of the technique of the spontaneous formation of a consistent structure. 
The section "Experience in Applying Spontaneous Structuring Technique" gives brief overview of technology application.

In section "Human Factor or the Role of an Expert in Using the Technology" the authors emphasize the role of a man as an expert when using the spontaneous formation technology of a consistent structure. The conclusion contains possible applications of the technology.

Thus, technology development (conception, algorithm and software) for consistent structure formation in the form of a set of the non-crossing stable multidimensional homogeneities (contiguity associations) that represents regions of adequate extrapolation (forecasting) of objects in them.

Keywords: forecast, structure, technique.

\section{References}

1. Dorodnitsyn A.A., Kaspshitskaya M.F., Sergienko I.V. On an approach to the formalization of classification. Cybernetics. 1976, no. 6, pp. 132-140 (in Russ.).

2. Ostrovsky E.Ya. Radioactive anomalies as indicators of the ore process. Reports of the USSR Academy of Sciences. 1975, vol. 221, no. 1, pp. 201-204 (in Russ.).

3. Portnov A.M. On the geochemical connection of potassium and thorium in igneous rocks and near-ore metasomatites. Reports of the USSR Academy of Sciences. 1978, vol. 239, no. 6, pp. 1441-1444 (in Russ.).

4. Ostrovsky E.Ya., Portnov A.M. Indicator properties of potassium and thorium geochemical connection. Geochemistry. 1979, no. 5, pp. 767-774 (in Russ.).

5. Portnov A.M. Radiogeochemical ore search. Nature. 1984, no. 5, pp. 99-105 (in Russ.).

6. Ostrovsky E.Ya., Rumyantsev S.V. Spontaneous structuring of digital geophysical images of natural complexes; forecasting of minerals. DAN. Geophysics. 2004, vol. 398, no. 3, pp. 396-399 (in Russ.).

7. Ostrovsky E.Ya., Rumyantsev S.V., Fadeev V.A. New structural analysis of digital images of natural complexes. Geoprofi. 2005, no. 2, pp. 49-51 (in Russ.).

8. Ostrovsky E.Ya. Asymptotic prediction based on remote geophysical studies. Reports of the USSR Academy of Sciences. 1986, vol. 289, no. 4, pp. 827-836 (in Russ.)

9. Gonzalez R., Woods R. Digital Image Processing. Moscow, Technosphere Publ., 2005, 1072 p.

10. Gonzalez R., Woods R., Eddins C. Digital Image Processing in the MATLAB Environment. Moscow, Technosphere Publ., 2006, 621 p. $280 \mathrm{p}$

11. Glensdorf P., Prigogine I. Thermodynamic Theory of Structure, Stability and Fluctuations. Moscow, Mir Publ., 1973,

12. Feder E. Fractals. Moscow, Mir Publ., 1991, 254 p.

13. VoxelGeo. Volumetric Interpretation and Allocation of Bodies. Available at: http://www.pdgm.com/products/voxelgeo/ (accessed April 2, 2018).

\section{Примеры бибциографического описания статьи}

1. Островский Э.Я., Румянцев С.В., Фадеев В.А. Развитие алгоритма спонтанного структурирования цифровых изображений природных комплексов // Программные продукты и системы. 2018. T. 31. № 3. C. 495-499. DOI: 10.15827/0236-235X.123.495-499.

2. Ostrovsky E.Ya., Rumyantsev S.V., Fadeev V.A. Development of the spontaneous structuring algorithm of natural complex digital images. Software \& Systems. 2018, vol. 31, no. 3, pp. 495-499 (in Russ.). DOI: 10.15827/0236-235X.123.495-499. 